\title{
Comment exercer une autorité experte ? Un scientifique confronté aux Sceptiques
}

How to Exercise Expert Authority: A Case Study of a Scientist Facing The Sceptics

Jean Goodwin

Translator. Marianne Doury

\section{OpenEdition}

Journals

Electronic version

URL: http://journals.openedition.org/aad/2035

DOI: $10.4000 /$ aad.2035

ISSN: 1565-8961

Publisher

Université de Tel-Aviv

\section{Electronic reference}

Jean Goodwin, «Comment exercer une autorité experte? Un scientifique confronté aux Sceptiques », Argumentation et Analyse du Discours [Online], 15 | 2015, Online since 15 October 2015, connection on 23 September 2019. URL : http://journals.openedition.org/aad/2035 ; DOI : 10.4000/aad.2035

This text was automatically generated on 23 September 2019

\section{cc) (ㅇ) $\odot$}

Argumentation \& analyse du discours est mis à disposition selon les termes de la licence Creative Commons Attribution - Pas d'Utilisation Commerciale - Pas de Modification 4.0 International. 


\section{Comment exercer une autorité experte? Un scientifique confronté aux Sceptiques}

How to Exercise Expert Authority: A Case Study of a Scientist Facing The Sceptics

Jean Goodwin

Translation : Marianne Doury

1 La théorie de l'argumentation n'est pas l'affaire exclusive des théoriciens de l'argumentation; ceux qui pratiquent l'argumentation théorisent également. Toute performance argumentative est sous-tendue par l'idée que celui qui argumente se fait de l'activité à laquelle il se livre. Les argumentateurs étiquettent certains procédés discursifs, sur la base de catégorisations qui peuvent être thématisées, contestées et justifiées ; et ces justifications peuvent à leur tour devenir objets de discussion. Ainsi, à la suite de Craig (1996), on considérera que théorie et pratique relèvent d'un même continuum: la théorie connaît divers degrés d'explicitation, et peut sous-tendre implicitement les échanges dans une interaction quotidienne ou en être extraite jusqu'à devenir un objet de discours en soi. Par exemple :

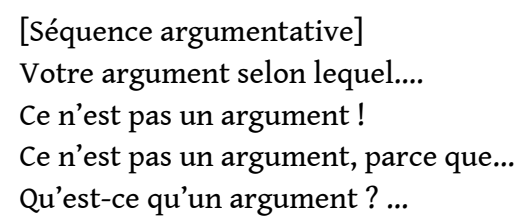

2 L'intérêt pour le savoir pratique des argumentateurs - leurs "folk theories" de l'argumentation (ou "argumentologie populaire », Doury 2013a) - constitue un point de convergence entre théoriciens de l'argumentation de la sphère francophone et de l'Amérique du Nord anglophone. Plusieurs auteurs, de part et d'autre de l'Atlantique, ont contribué à une meilleure compréhension des concepts partagés par les théoriciens et les praticiens de l'argumentation, et notamment, des concepts même d' " argument " ou d'« argumentation» (Craig 2011, Doury 2008, Goodwin 2007, Plantin 1996), de 
l'anglais issue (Craig \& Tracy 2005, Goodwin 2002), de la "charge de la preuve " (Kauffeld 2009), ainsi que de types d'arguments ou de paralogismes spécifiques (Doury 2010a). Ces travaux, qui adoptent une approche empirique de l'argumentation, cherchent à rendre compte de cas complexes et s'attachent à élucider la façon dont les praticiens eux-mêmes se représentent leur activité, à travers notamment le métadiscours des argumentateurs (Craig 1999, Doury 2013a, Plantin 1996).

Dans cet article, j'avance une suggestion qui peut être considérée comme radicale : les théoriciens de l'argumentation doivent avant tout rendre justice aux savoirs que les argumentateurs déploient et thématisent dans les interactions argumentatives ordinaires. Pourquoi cela? En premier lieu, parce qu'il y a plus de praticiens que de théoriciens de l'argumentation. Il se pourrait que les premiers soient plus motivés que les seconds (ils veulent l'emporter), et qu'ils soient également plus malins (ils n'ont pas décidé de devenir des théoriciens de l'argumentation). Mais la raison principale est que les interactions argumentatives sont pour partie déterminées par les conceptions que les participants ont de l'activité à laquelle ils se livrent. Les questions envisagées à un niveau abstrait par les théoriciens de l'argumentation se posent comme des problèmes pratiques qui nécessitent une résolution immédiate pour les argumentateurs. Les solutions que ces derniers sont amenés à mettre en œuvre doivent «marcher ». Elles doivent être acceptées (ou du moins, ne pas être rejetées) par les autres participants à l'interaction ${ }^{1}$, elles doivent contribuer à la co-construction et à la gestion de l'interaction.

4 L'étude de cas que je présente ici est centrée sur l'appel à l'autorité experte. D'un point de vue théorique, ce procédé a été souvent étudié et enseigné. Le traitement adéquat de la délégation d'expertise est même une préoccupation centrale pour la théorie de l'argumentation, et de nombreux manuels font place à la question de l'argument (ou du paralogisme) de l'appel à l'opinion experte. Dans la pratique, les questions sociotechniques ont acquis une forme d'hégémonie dans les délibérations publiques, ce qui amène à s'interroger sur la façon dont, en pratique, le discours expert peut être intégré à des échanges citoyens. Le changement climatique, les organismes génétiquement modifiés, les technologies de procréation humaine, l'énergie nucléaire : tous ces sujets ont plongé les scientifiques dans des controverses publiques brûlantes, soulevant de nouveaux problèmes pratiques que les argumentateurs doivent résoudre.

5 Le talk-show «The Sceptics » du 7 septembre 2010, qui a organisé la rencontre entre le spécialiste du climat Stephen H. Schneider et un panel de 52 sceptiques, constitue un excellent terrain pour observer la façon dont les argumentateurs conçoivent l'autorité experte. En 1971, année où Schneider a soutenu sa thèse de doctorat, les recherches menées par son équipe suggérant que la terre est menacée par un changement climatique catastrophique (c'est, à cette époque, à un refroidissement climatique que l'on s'attendait) ont attiré l'attention des médias. En tant que plus jeune membre de l'équipe, Schneider était chargé d'en assurer les relations publiques. Il a pris goût aux interactions avec les médias, et y a excellé. Aussi au cours des quarante années qui ont suivi Schneider a-t-il poursuivi sa carrière sur un triple front: une recherche scientifique d'excellence, la participation à des dispositifs de consultation scientifique comme le Panel Intergouvernemental sur le Changement Climatique (IPCC), et une activité soutenue de diffusion de la science auprès du grand public. Outre de nombreuses apparitions dans des émissions télévisées de divertissement ou des émissions plus sérieuses, des tribunes dans la presse écrite ou des conférences 
publiques, il a publié plusieurs ouvrages sur le changement climatique destinés au grand public. Ces efforts lui ont valu une récompense de l'American Association for the Advancement of Science, ainsi que le "prix des génies » de la fondation Mac Arthur. Menant une réflexion sur sa propre pratique, Schneider a aussi beaucoup écrit sur les défis posés par la communication scientifique dans des contextes de controverse ${ }^{2}$. Ses expériences antérieures comme membre étudiant d'un conseil d'université pendant la guerre du Vietnam l'avaient amené à " reconnaître l'existence de différents systèmes de valeur, puis à chercher à fonder des valeurs communes permettant à tous de vivre ensemble (Martin 2010). Il affirme avoir "détesté » son rôle de «ferrailleur scientifique », mais a toutefois apprécié « un débat ouvert et honnête », même avec des interlocuteurs profondément dubitatifs (Snell 2009).

6 En 2010, alors que Schneider était en Australie pour une conférence, il a été sollicité pour intervenir dans une émission télévisée consacrée au sujet de plus en plus clivant $\mathrm{du}$ changement climatique. L'Australie est considérée comme particulièrement vulnérable au réchauffement climatique, avec des températures en hausse et des sécheresses de plus en plus fréquentes favorisant les feux de forêt et les pénuries d'eau. Dans le même temps, l'Australie a un taux relativement élevé d'émission de carbone par habitant, et une industrie du charbon importante et politiquement influente. Au moment de sa visite, la politique climatique était un sujet d'affrontements majeur, le gouvernement rencontrant une opposition virulente à ses propositions d'échange de quotas d'émission et de taxe carbone. C'est dans ce contexte que Schneider a été invité à participer à "Insight », une émission bien ancrée à la télévision australienne, animée par Jenny Brockie, journaliste dont le travail a été salué par de multiples récompenses. Cette émission fait dialoguer pendant une heure des experts et des citoyens ordinaires sur un sujet spécifique. Schneider a accepté de participer à cette émission "parce que les scientifiques doivent aussi s'efforcer d'aider les gens à comprendre le risque. Et si vous êtes en permanence dans une logique de combat, comment voulez-vous que l'on puisse communiquer de façon citoyenne? " C'est ainsi qu'au mois de juin, Schneider s'est retrouvé dans un amphithéâtre universitaire, face à 52 individus se qualifiant euxmêmes de « climato-sceptiques".

7 Cet événement a conféré une publicité accrue à l'expertise de Schneider, dans des conditions optimales - du moins au regard des contraintes imposées par le monde réel. Son décès inopiné un mois plus tard amène à voir cette émission comme l'ultime prestation d'un communicant hors normes. Tous ces éléments nous autorisent à considérer le discours de Schneider dans ce contexte comme un exemple de bonne pratique argumentative, qui peut constituer un cas paradigmatique d'exercice approprié d'une autorité.

8 Dans ce qui suit, je proposerai tout d'abord une reconstruction de la façon dont les participants comprennent le cadre social dans lequel ils se trouvent; j'identifierai ensuite deux stratégies majeures utilisées par les participants pour gérer les désaccords qui les opposent ${ }^{3}$. Je conclurai sur une comparaison entre les conceptions que se font théoriciens et praticiens de l'autorité experte.

\section{Le contexte social de l'appel à l'autorité}

9 L'émission s'ouvre sur une brève introduction du thème du débat par l'animatrice : 
A : le changement climatique généré par l'activité humaine a été une préoccupation majeure pour la société, mais l'intérêt politique qu'elle a suscité a décru alors que grandissait un sentiment de scepticisme dans le grand public. Ce soir, un auditoire de sceptiques rencontre un des plus grands climatologues au monde, qui va chercher à les gagner à sa cause.

L'animatrice invite alors certains membres du public à présenter brièvement leur position; chacun exprime des doutes sur l'existence d'un réchauffement climatique d'origine humaine. S'ouvre ensuite une succession d'échanges nourris entre Schneider et certains sceptiques sur des points précis, parmi lesquels, l'intensité du réchauffement récent, la variabilité à long terme du climat, l'attribution du changement climatique à l'activité humaine, la fiabilité des mesures de température, et l'impact du changement climatique sur les glaciers. Chacun de ces sujets est récurrent dans le débat général sur le climat; Schneider était bien préparé pour les aborder, et l'animatrice lui a ménagé d'assez longues plages de parole pour les traiter. A un peu plus de la moitié de l'émission, la discussion s'engage à un niveau " méta ", sur la façon même dont le changement climatique est abordé : l'expression de l'incertitude dans les rapports de l'IPCC, l'excessive polémicité et la politisation du débat, l'incapacité des scientifiques spécialistes du climat à maintenir une distance suffisante vis-à-vis des militants environnementalistes, le rôle joué par les médias dans la distorsion de la science, et l'évaluation de la crédibilité des références sur le sujet. L'émission s'achève par l'intervention d'un membre du public invitant Schneider à donner sa position personnelle sur la question du climat. Au total, 15 des 52 membres du public ont pris la parole, et 7 d'entre eux ont participé à plus d'un échange.

11 Ainsi que le suggère le nom même de l'émission, l'interaction est conçue comme organisant la confrontation de Schneider à des "Sceptiques". Mais comment les participants se présentent-ils et se reconnaissent-ils mutuellement? Pour sa part, Schneider se positionne comme s'exprimant pour ses "collègues", qu'il désigne comme "climatologues", "scientifiques spécialistes du climat ", "l'IPCC », ou plus généralement, par les termes «scientifiques» ou «communauté scientifique». Deux des membres du public, par contraste, s'identifient explicitement comme «non scientifiques ", sans pour autant expliciter positivement ce qu'ils sont :

Katy Barnett: Je ne suis pas une scientifique, je ne peux exprimer aucune opinion sur la science.

A : Vous êtes avocate.

Katy Barnett : je suis avocate, alors vous pouvez m'attaquer en justice.

$\mathrm{A}$ : Mais vous êtes la fille de deux scientifiques, n'est-ce pas, et vous êtes mariée à un scientifique.

Katy Barnett : Eh bien oui.

SS : Alors vous êtes bien entourée.

Jessica Berry : ...ceux d'entre nous qui tiennent à aller au-delà des cinq secondes qu'on nous propose au journal du soir mais qui ne sont pas des scientifiques, qui sont juste des monsieur-tout-le-monde...

D'autres membres du public s'identifient implicitement comme non scientifiques en se distinguant (« je / nous ») du groupe des scientifiques (« vous / eux ») :

David Clark: oui, vous nous servez votre baratin pour les médias, mais je pense qu'en tant que sceptiques, en tant que profanes, en tant que membres ordinaires du public, il y a assez d'information disponible pour qu'on trouve ce qu'on veut si on le souhaite.

Chris MacDonald: eh bien, c'était très intéressant de vous entendre parler tout à l'heure sur, sur comment les scientifiques ont plutôt une position médiane, modérée, 
oui, modérée. Ce qui me semble louche, c'est que je n'ai entendu aucun... aucun de ces scientifiques modérés s'exprimer pour faire un sort aux scénarios apocalyptiques que nous débitent les environnementalistes et nos politiciens. Je ne parle pas de vous personnellement, monsieur, mais votre industrie, vos lobbys, le lobby auquel vous appartenez... J'aimerais entendre des gens de votre business reconnaître qu'il y a des doutes... je n'en ai jamais entendu aucun se lever pour dire... je n'ai jamais entendu un scientifique du IPCC dire ça.

Tania Berman : La seule raison pour laquelle vous êtes financé, c'est le changement climatique, la planète se réchauffe, c'est la seule raison pour laquelle vous touchez des financements. ${ }^{4}$

Dans ces extraits, les scientifiques sont présentés comme un groupe défendant ses propres intérêts (« industrie, lobby, business »), faisant usage de stratagèmes politiques typiques («baratin pour les médias») à leur propre profit (pour «toucher des financements »). Les « membres ordinaires du public », les « monsieur-tout-le-monde » sont témoins de cette attitude.

Ces deux groupes - que je désignerai par "les scientifiques" et "les profanes" - se distinguent aussi par leur façon de parler du changement climatique. Considérons en premier lieu le discours profane. L'animatrice ouvre la discussion en demandant aux membres du public: "croyez-vous au changement climatique généré par l'activité humaine? ». Plusieurs profanes répondent alors en marquant systématiquement leur position par des modalisateurs du type « je crois / je ne crois pas ». Par exemple :

Sc: euh, je ne suis pas particulièrement sceptique, mais je crois que, euh, le changement climatique c'est, euh, quelque chose qui, qui se produit naturellement dans euh... dans le monde. Mais je pense que ça peut être produit par l'humanité, dans le sens que ce que fait l'humanité peut l'accélérer. Donc je ne crois pas que le réchauffement climatique, que le réchauffement climatique soit produit par l'homme, je pense que c'est naturel et que c'est, ça rentre dans un processus climatique

Ici, « je pense / crois que » introduit chacune des assertions - assertions qui sont ellesmêmes avancées avec une incertitude dont témoignent les hésitations, les corrections ainsi que certains choix lexicaux inhabituels. Un seul membre du public (on y reviendra) rejette l'invitation de l'animatrice à dire « ce qu'il croit ».

Par contraste, l'animatrice oriente tout autrement la réponse de Schneider en l'invitant à exprimer non pas ses "croyances", mais à énoncer «l'argument le plus fort qu'il pourrait opposer à ces gens ». Schneider prend alors la parole en rejetant tout discours en « je crois» :

SS : j'ai entendu les mots «je ne crois pas». La science n'est pas une affaire de croyance, la science est une affaire de preuves, et pour vous forger une conviction, vous devez regarder un large éventail de preuves.

17 Le paradigme visuel du regard est présent dans l'ensemble de son discours ; il décrit les scientifiques comme "observant» des périodes aussi longues et anciennes que le "passé géologique", ou aussi limitées et récentes que "les cinquante dernières années », des espaces étendus "à la planète entière » et un " large éventail de preuves " dont des indices aussi ténus que les "anneaux de croissance des arbres» et le "pollen ». Dans son évocation de ce que les scientifiques ont observé, le discours de Schneider est remarquablement dépourvu de modalisations, à l'exception occasionnelle d'un «nous savons que » ou «en réalité ${ }^{5}$. Les assertions catégoriques sur le monde sont la norme, comme dans l'exemple suivant : 
SS : Il y a eu un important réchauffement statistique au cours des euh des quinze dernières années. La discussion porte sur le fait de savoir si ce réchauffement est "statistiquement significatif», comme on dit, ce qui impose des conditions draconiennes aux statistiques recueillies. En fait, durant... euh, en quelle année sommes-nous, durant la première moitié de 2010, on a enregistré les températures les plus chaudes jamais mesurées. Et ce qui s'est passé, c'est que de 1992 à environ 2002, la température a augmenté si vite qu'on aurait pu croire qu'on filait droit à la catastrophe. Puis de 1998 à environ 2009, elle s'est relativement stabilisée. porté par Schneider : la quantification, et ce qu'un des membres du public appelle le «jargon technique ».

\section{Une première réponse à l'opposition « scientifiques / profanes » : une invitation à rejoindre la communauté scientifique}

19 Ainsi, l'interaction étudiée met en présence deux groupes, caractérisés par des pratiques discursives spécifiques: des profanes qui expriment leurs croyances, des scientifiques qui observent des preuves et rapportent leurs découvertes. Comment ces deux groupes sont-ils supposés interagir?

La première approche adoptée par Schneider pour dépasser cette répartition des participants en deux camps et réduire la distance entre scientifiques et profanes consiste en ce qu'on peut appeler une stratégie d'invitation. Il réalise cet objectif en suivant le conseil classiquement prodigué par les manuels de communication scientifique, et dans ses propres rêveries sur la "médiarologie » :

Je m'efforce d'adopter un langage et des métaphores accessibles. Le jargon scientifique est adapté à la communication entre scientifiques, mais est source de malentendus dans l'arène publique, et augmente la probabilité qu'un scientifique soit "catalogué ", que ses propos soient déformés ou tout simplement ignorés. A mes yeux, les métaphores qui évoquent à la fois l'urgence et l'incertitude sont les meilleures - en particulier pour traiter des cas controversés, comme les conséquences irréversibles du changement climatique (Schneider 2009 : 230).

On a vu, dans l'exemple précédent, comment Schneider, lorsqu'il utilise un terme technique, signale explicitement sa présence («statistiquement significatif », comme on dit), et interrompt son développement pour expliquer ce qu'il signifie. Ailleurs, il utilise des "métaphores "- ou, plus précisément, des analogies; il compare ainsi le déséquilibre du cycle carbone à une baignoire qui se remplirait plus vite qu'elle ne se viderait. Ces procédés permettent aux profanes d'avoir accès aux discussions entres scientifiques, du moins sous une forme simplifiée.

En complément de ces efforts pour élever les profanes jusqu'aux sommets du langage scientifique, Schneider cherche à dépasser cette division en deux groupes sociaux. Il salue les tentatives des profanes pour adopter une attitude scientifique. Il qualifie de "parfaitement légitime" la démarche qui consiste à "prendre en considération une longue période de l'histoire »- démarche suggérée par «tout le monde » au cours des tours précédents. De même, il ouvre souvent ses interventions en félicitant son interlocuteur de poser des questions (voir Goodwin, à paraître) - activité de discours qu'il caractérise à plusieurs reprises comme typiquement scientifique. Par exemple :

SS: oui, c'est une bonne question, c'est ainsi que procède la communauté scientifique... Et l'excellente question que vous avez posée est en tout point 
similaire à celle que les scientifiques spécialistes du climat se posent depuis trente à quarante ans.

Il en vient même à s' " interrompre une seconde " pour établir explicitement une équivalence entre scientifiques et profanes :

SS : Je me réjouis vraiment que vous soyez sceptiques. Un bon scientifique qui ne serait pas sceptique, ça n'existe pas.

Dans le même temps qu'il élève son auditoire au niveau de la science, Schneider s'efforce de se mettre au niveau des profanes en suggérant qu'il adhère aux mêmes valeurs qu'eux, et qu'il partage leurs préoccupations. Il insiste sur le fait que comme les membres du public, il regrette le manque de courtoisie qui règne dans les discussions sur le changement climatique. Après que trois d'entre eux ont raconté le harcèlement qu'ils ont subi après avoir exprimé leurs doutes sur le changement climatique, il produit un témoignage en miroir, rapportant qu'il a lui-même reçu des menaces de mort et autres mails déplaisants, y compris un message adressé le jour même à son fils. Et il conclut la rencontre en répondant à un membre du public, Jessica Berry, qui lui avait demandé :

Il me semble que ça pourrait être une bonne chose de simplifier tout le jargon et de mettre les choses dans une perspective que tout le monde peut comprendre. Avec ce que vous savez, et après avoir étudié, comme vous l'avez dit, pendant quarante ans, est-ce que vous avez peur ? Est-ce que vous avez peur pour vos petits-enfants?

Cette question est providentielle pour Schneider, dans la mesure où elle lui permet d'endosser le rôle d'un individu ordinaire (qui aurait des "petits-enfants ») pour exposer sa propre évaluation de la question des risques, et exprimer ses propres peurs.

Certains indices suggèrent que la stratégie d'invitation de Schneider a marché - du moins auprès d'une partie du public. Vers la fin de l'émission, la sceptique Katy Barnett le remercie "d'avoir participé à un dialogue en restant raisonnable et sans fondamentalement, sans diaboliser ceux qui osent exprimer un doute ». Et quelques personnes lèvent la main lorsque l'animatrice demande si certains ont changé d'avis.

Mais d'autres indices amènent à penser que cette stratégie d'invitation a aussi ses limites. Plusieurs profanes - en particulier, ceux qui s'étaient présentés comme non scientifiques - déclinent l'invitation. David Clark, dont on a reproduit plus haut l'autocatégorisation comme "profane ", souhaite insister sur ce qui constitue, pour lui, la vraie question :

Et pourquoi on vous croirait vous, plutôt que votre voisin? Là, on n'a aucun scientifique qui représenterait l'autre camp, parce que ça aurait probablement rendu impossible de mener à bien l'émission... Et on se retrouve avec ce genre de chamailleries... Pourquoi on vous croirait vous plutôt qu'un autre, alors que des deux côtés, on a des arguments crédibles?

Ce profane n'accepte pas l'invitation de Schneider à se joindre à la discussion entre scientifiques. Il s'en tient au discours ordinaire de la «croyance », conçue ici comme croyance en une personne plutôt qu'à un fait. D'autres membres du public prolongent sa question en expliquant les raisons pour lesquelles ils ne croient pas les scientifiques : parce que les scientifiques sont partiaux, parce que les scientifiques n'énoncent pas de certitudes, parce que les scientifiques ne présentent qu'un côté de l'affaire, parce que les scientifiques ne contestent pas les affirmations extrêmes ou alarmistes - à l'inverse, eux-mêmes avancent des affirmations alarmistes. Deux des profanes les plus loquaces justifient expressément leur scepticisme par des raisons non scientifiques. 
Janet Thompson: Je pense qu'on a là un problème de fond, on veut changer complètement la structure de notre économie sur la simple hypothèse que c'est le $\mathrm{CO} 2$ qui décide du climat.

Tania Berman : ça augmente juste un peu, et pourtant, on veut changer toute notre économie sur la base de cette petite augmentation [de $\mathrm{CO} 2$ ] qui en fait nous arrange bien.

\section{David Clark :}

Très bonne question. J'assure un séminaire d'initiation à l'environnementalisme pour des étudiants de première et deuxième année, et comment peut-on discerner la qualité d'un argument? Il faut prendre garde aux traqueurs de mythes et aux diseurs de vérités. Ceux qui pensent avoir la réponse à un problème complexe ne sont pratiquement jamais dignes de foi. Ceux qui, lorsqu'ils parlent, passent par des circonvolutions, empruntent des détours et font une part au hasard - et oui, je prêche pour ma chapelle, je suis de ceux-là - et c'est à cause de la nature même de la compréhension du monde par la science - et c'est le cas aussi de l'IPCC. Alors il me semble que le meilleur critère, pour vous, c'est que, devant un problème complexe, il faut se rappeler qu'on doit essayer de le décomposer en petits bouts bien établis où on a des éléments qu'on peut considérer comme très probables; il faut prendre en compte des explications concurrentes - par exemple, le Groenland est en train de fondre [plus rapidement que prévu], mais pourquoi ça se passe comme ça, ça, on ne sait pas; et certains points restent spéculatifs - on n'a pas vraiment idée de l'importance que va avoir la rétroaction nuageuse. Et quand les gens parlent comme ça, il y a bien plus de chance qu'ils soient crédibles que quand ils vous disent qu'ils ont, vous savez, la preuve décisive qui va départager ceux qui ont raison et ceux qui ont tort. C'est très rarement le cas, sur les questions scientifiques complexes.

Cette réponse peut étonner. On distingue les scientifiques des profanes sur la base de leur capacité à énoncer des assertions catégoriques sur le monde, plutôt qu’à livrer leurs «convictions». Mais Schneider incite les profanes à accorder plus de confiance aux assertions moins catégoriques. C'est là la première caractéristique de l'exercice de l'autorité : la modestie dont fait preuve le scientifique dans ses affirmations. 

parce qu'elle rend compte plus fidèlement des résultats scientifiques. Bien qu'on dispose de " petits bouts » « bien établis ", Schneider explique qu'en général, quand un sujet est "compliqué / complexe», on ne peut pas avancer de réponses catégoriques. Selon cette logique, les assertions modestes sont susceptibles de mieux refléter l'état réel de la science. Schneider ouvre deux autres pistes articulant modestie et fiabilité d'un scientifique. Toutes deux mettent l'accent sur des aspects normatifs du discours du scientifique.

En premier lieu, les scientifiques ont le devoir de représenter fidèlement la science dans sa complexité ; cela relève de leur responsabilité. C'est ce qu'explique Schneider en réponse à Chris MacDonald, cité plus haut, qui regrette n'avoir jamais entendu aucun scientifique exprimer un doute :

S'il vous plaît, lisez mon livre, vous verrez que ça fait quarante ans que je m'y efforce. Et je ne suis pas le seul à faire ça. Je pense que ça serait irresponsable de notre part d'exclure les scénarios les plus optimistes, de même qu'il serait irresponsable d'écarter les pires. Ce n'est pas notre boulot, en tant que scientifique, de décider si les risques sont suffisants pour se prémunir contre ces possibilités. Notre boulot, ça se limite à rendre compte des risques... En ce qui me concerne, je peux dire que c'est exactement ce que je fais. Mais je peux aussi vous dire - et avec moi, bon nombre des collègues que je respecte - que ce n'est pas le cas de tout le monde. Il y a des gens qui minimisent, et des gens qui exagèrent.

Ainsi, la modestie scientifique est plus crédible parce qu'elle manifeste que le scientifique lui-même respecte des normes communicationnelles. Au-delà du fait qu'il y a des chances que ce qu'il dit soit plus fidèle, le scientifique lui-même apparaît comme personnellement digne de confiance. La crédibilité a donc un coût : quelle que soit la position vers laquelle il penche en son for intérieur - aussi assuré qu'il se sente - en public, le scientifique doit ostensiblement assumer la responsabilité de limiter la portée de ses assertions.

Une seconde norme, qui exige elle aussi de la modestie de la part des scientifiques - et plus largement, des citoyens - est liée à la volonté de favoriser des délibérations de qualité sur des questions controversées. En réponse à une question sur la crédibilité des ressources en ligne, Schneider répond :

Je suis très vite gêné par les blogs elliptiques, ceux qui sont vraiment polarisés dans un sens ou dans l'autre, parce qu'on - généralement, ils sont le fait de très bons écrivains, et si quelqu'un - si quelqu'un y croit déjà ils sont très intéressants, mais là encore, ce qu'on perd, c'est un dialogue citoyen.

Schneider utilise l'adjectif "elliptique» dans d'autres contextes pour désigner les approches qui rejettent la science officielle; il active ainsi son sens mathématique de " déformé » ou " décentré », ou son sens grammatical, pour qualifier une approche où quelque chose - la complexité - est passé sous silence. Schneider considère également que la tendance qu'ont les médias à faire fi des nuances et à présenter tout désaccord comme un affrontement entre deux positions extrêmes (l'une voyant le changement climatique comme la "fin du monde», et l'autre comme «bon pour vous») «tend à polariser» le débat. Dans cette perspective, pour apparaitre digne de confiance, le scientifique doit ostensiblement se plier aux normes du "dialogue citoyen», en présentant notamment avec modestie l'ensemble des résultats scientifiques.

38 Les sources que Schneider mentionne comme crédibles respectent le principe de modestie: les rapports de l'IPCC, par exemple, qu'il décrit comme "truffés de 
réserves ». Plus significatif encore, Schneider affirme avec insistance qu'il respecte luimême ces règles. Cette affirmation, que l'on trouve dans plusieurs des exemples précédents, revient dans ses contributions tout au long de l'émission. Il admet à plusieurs reprises que les méthodes ont des limites ( il y a des problèmes avec les instruments»), que les résultats varient (le réchauffement décennal a connu des fluctuations, les températures "sont différentes en des points différents" du Groenland), que les théories débouchent sur des prédictions erronées (les glaciers fondent « plus rapidement que prévu par aucune théorie »), et que parfois, « on n'a tout simplement pas de données ». Il présente la communauté scientifique comme parfois divisée, notant par exemple que l'interprétation des anneaux de croissance des arbres est controversée, et qu' «il y a souvent une tension intéressante entre ceux qui élaborent les modèles et font de la théorie et ceux qui mesurent, ces derniers tendent à être un peu plus sceptiques». Dans sa dernière intervention, où il exprime sa préoccupation, il commence par évoquer la «probabilité très inconfortable" d'« évolutions plutôt déplaisantes » (10\%), pour évoquer immédiatement la probabilité «qu'il n'y ait pas énormément d'évolutions déplaisantes », voire pas du tout («10,20\% de chances »).

A l'opposé de la figure du pourvoyeur de science modeste donc crédible, on trouve les « traqueurs de mythes et les diseurs de vérités » qui prétendent " avoir LA réponse » la "preuve décisive qui va départager ceux qui ont raison et ceux qui ont tort». Bien qu'aucun des interlocuteurs de Schneider n'admette être un "diseur de vérités ", on peut noter que plusieurs d'entre eux empruntent le langage de la "preuve » et de la « réfutation ». Par exemple:

Tania Berman : Personne ne m'a prouvé que c'était d'origine humaine

Sc: Maintenant l'IPCC considère que les exemples de glaciers qui fondent ne constituent pas des preuves suffisantes de l'effet du réchauffement climatique

Ces exemples suggèrent que les locuteurs qui les énoncent attendent des scientifiques qu'ils leur procurent de la certitude, et exigent d'eux des affirmations immodestes. A l'inverse, Schneider, pour sa part, n'utilise le langage de la "preuve » qu'à une reprise, qualifiant de "ridicule» l'affirmation selon laquelle il existerait un ensemble de mesures de la température qui « réfuterait le réchauffement climatique ».

41 Telle est la première face de l'exercice de l'autorité : pour inspirer confiance, un scientifique doit ostensiblement assumer ses responsabilités en limitant la portée de ses affirmations et en admettant avec toute la modestie requise les incertitudes, les controverses, et le caractère purement spéculatif de certaines conjectures. Même un étudiant de première année qui suivrait les cours de Schneider est en mesure d'utiliser ces indices pour identifier quels scientifiques sont crédibles et quels scientifiques le sont moins. Mais l'exercice de l'autorité a une seconde face. Les profanes ont euxmêmes des limites lorsqu'ils interagissent avec les scientifiques - limites que les scientifiques ont le droit de relever. A trois reprises dans ses échanges avec les Sceptiques, Schneider conteste ce que son interlocuteur profane affirme. Chaque fois, le profane avait tenté de parler comme un scientifique; chaque fois, Schneider lui oppose qu'il « a tort ».

Le premier exemple se produit lors du premier échange soutenu de la rencontre, avec un membre du public qui commence par reprendre à son compte l'affirmation de Schneider que « la science n'est pas une question de croyance » : 
Janet Thompson: Eh bien, je me réjouis que le Professeur Schneider ait commencé en disant «il faut regarder les preuves ». Et je ne pense pas que qui que ce soit ici contesterait cela. L'hypothèse à laquelle nous sommes confrontés actuellement est que le dioxyde de carbone est l'agent du changement climatique. Et tout au long de l'histoire, on a recueilli des preuves démontrées que les températures ont été considérablement plus basses qu'aujourd'hui, avec des taux supérieurs de $\mathrm{CO} 2$ dans l'atmosphère, et inversement. Pouvez-vous -

A : Est-ce que je peux juste vous demander, Janet, pensez-vous que la Terre soit réellement en train de se réchauffer? Croyez-vous en, que le réchauffement est réel, indépendamment de sa cause?

Janet Thompson: Eh bien, je ne crois pas quoi que ce soit, je regarde les preuves et les preuves disent qu'un réchauffement s'est produit, oui, on a connu une séquence de réchauffement de longue durée durant les quinze dernières années, on n'a pas connu de réchauffement stastistique [sic]. Et donc je pense qu'il y a un problème avec cette hypothèse. Je, je crois que cette hypothèse a été réfutée.

SS : oui, ok, c'est faux, désolé - ce n'est pas ce que montrent les preuves. D'abord...

Thompson - qui bénéficie d'un temps de parole supérieur à tous les autres participants, à l'exception de l'animatrice et de Schneider lui-même - s'efforce clairement de parler comme une scientifique. Elle refuse explicitement de répondre à l'incitation de l'animatrice d'exprimer "ce qu'elle croit». Elle choisit plutôt d'avancer ce qu'elle appelle une " hypothèse ", " regarde les preuves", cherche à utiliser un vocabulaire technique («l'agent du changement climatique», " réchauffement statistique »), et énonce une série d'affirmations sur le monde. Ses tentatives pour parler comme une scientifique sont maladroites; au début de son intervention, elle réclame des «preuves démontrées ", elle peine à caractériser correctement la séquence de réchauffement récente, et au terme de son intervention, elle passe à un discours en "je crois ». Mais loin de saluer ses efforts pour se rapprocher de la communauté scientifique en énonçant un de ses «bonne question!» habituels, Schneider rejette sa tentative. Il adoucit son rejet en ouvrant son intervention par des marques de validation " oui, ok ", et en recourant à un procédé d'indirection (« c'est faux » plutôt que « vous avez tort »). Mais le sens de sa réaction est clair : Schneider affirme son droit à dire ce qui constitue un discours scientifique acceptable, et ce qui s'en démarque. discussion une troisième fois. Janet prend la parole alors que Schneider achève une explication sur l'acidification des océans, et objecte :

Janet Thompson: Mais c'est - ce que - la terminologie que vous utilisez est alarmiste. L'océan n'est pas acide, il est alcalin.

SS : oui, c'est-

Janet Thompson : Et ce dont vous parlez, c'est de la baisse d'un pH aux alentours de 8.2 à 8.1

SS : oui

Janet Thompson: Il y a donc beaucoup de discours catastrophistes qui circulent dans le monde. Et - tout à l'heure vous parliez d'une hausse d'environ $30 \% \mathrm{du} \mathrm{CO} 2$. L'impact du CO2 sur les températures est logarithmique - il n'est pas linéaire donc ces $30 \%$ ne signifient pas vraiment grand-chose dans la mesure où on a déjà connu des cas où le $\mathrm{CO} 2$ avait doublé au tout début des mesures de la température. Je suis donc préoccupée par l'alarmisme excessif qu'on a là, et par le vocabulaire qu'on emploie.

A : [inaudible]

SS : Est-ce que je peux juste répondre rapidement? Moi, ce qui me préoccupe, c'est de vous entendre répéter un mantra que vous avez entendu, reposant sur des informations dépourvues de toute crédibilité. 
Une nouvelle fois, Thompson parle comme une scientifique, recourant à des procédés de quantification et à un vocabulaire technique ("alcalin", "logarithmique"), dans des affirmations non modalisées. Et cette fois encore, Schneider rejette sa proposition, tout en adoucissant son rejet en utilisant un marqueur d'attitude poli («moi, ce qui me préoccupe ») qui fait écho à celui que vient d'utiliser Thompson, et en rejetant la responsabilité de son erreur sur des individus non identifiés qui l'auraient mal informée. Schneider poursuit par une explication de "l'absorption logarithmique » malgré les interruptions conjuguées de Thompson et de l'animatrice, et conclut :

Est-ce que je peux finir, s'il vous plaît ? C'est quelque chose qu'on connaît depuis 50 ans. Tout scientifique digne de ce nom utilise ce mode de calcul, avec un logarithme, dans n'importe quelle modélisation du climat. C'est tenu pour acquis. Et quand les gens commencent à dire que parce que le $\mathrm{CO} 2$ a une capacité d'absorption logarithmique, il ne peut pas avoir d'effet, soit ils ne comprennent rien à la climatologie, soit ils cherchent à polémiquer. Parce que c'est intégré à tous les modèles, ça a été pris en compte depuis longtemps, et c'est parfaitement élucidé.

Schneider réclame à nouveau, et avec plus de force encore, le droit à parler pour tous "les scientifiques dignes de ce nom», et à décider quelles sont les affirmations acceptables - un choix qu'il applique dans la dernière phrase, recourant à une structure ternaire ascendante classique : «c'est... ça a été... c'est... » ${ }^{6}$. Au minimum, «les gens » qui disent le contraire "ne comprennent rien à la climatologie». Au pire, "ils cherchent à polémiquer ", déforment intentionnellement la science et sapent toute discussion citoyenne afin de marquer des points dans leur stratégie politique. Dans tous les cas, ces "gens» (c'est-à-dire Thompson) voient leur participation au débat disqualifiée ; conclusion que l'animatrice valide implicitement en attribuant le tour de parole suivant à un autre membre du public.

Entre ces deux échanges apparaît une manifestation de la face « sévère » de l'autorité scientifique ; ce sera notre dernier exemple. Il commence de façon prototypique par des félicitations adressées par Schneider à un profane pour sa " question".

Ian Rivlin: Si je comprends bien, le dioxyde de carbone produit par l'homme représente $3 \%$ de ce que la nature produit. Comment de légers changements dans notre production de $\mathrm{CO} 2$ pourraient-ils avoir des effets à une échelle aussi grande que la planète? Ça semble absurde.

SS : oui, non, non seulement ce n'est pas absurde, mais c'est absolument vrai. Mais c'est une très bonne question, parce que ce qui se passe, c'est que...

Alors que l'explication de Schneider touche à sa fin, le profane l'interrompt :

Ian Rivlin: Désolé, désolé de vous interrompre là-dessus. Mais vous ne répondez pas à la question. J'ai dit qu'on produisait environ $3 \%$ de la production naturelle. Vous n'avez pas vraiment répondu à ça. Vous, vous avez éludé la question. Je veux dire SS : Peut-être que vous n'avez pas compris la réponse. Ce que j'ai dit, c'est que le taux...

Ian Rivlin : Non, j’ai parfaitement compris la réponse. Vous n'avez pas -

Après quelques tours de parole produits en chevauchements, Schneider commente :

SS : alors je ne comprends pas comment vous pouvez en venir à cette conclusion. Je, est-ce que ce que vous voulez dire, c'est que l'activité humaine ne peut pas être responsable de l'augmentation du dioxyde de carbone au cours du siècle dernier? Ian Rivlin : Absolument, c'est ce que je dis. Je dis qu'il y a homéoSS : Oh, alors vous avez complètement tort ${ }^{7}$.

Ian Rivlin : Alors je dis qu'il y a homéostase sur Terre SS : Non, vous avez complètement tort.

Ian Rivlin: Cette, cette petite production supplémentaire de dioxyde de carbone 
serait facilement absorbée.

SS : Non, je pense que vous devriez étudier cette question. Permettez-vous

Ian Rivlin : Je l'ai étudiée [inaudible]

A : Ok, ok

SS : De toute évidence pas correctement.

A : Chacun son tour

SS : Laissez-moi vous donner un exemple.

A : Laissez Stephen, laissez Stephen répondre.

SS : Si vous prenez une baignoire...

\section{L'animatrice lui ayant expliciten
longue explication et conclut :}

c'est parfaitement établi, ça a été établi depuis longtemps et si vous ne l'admettez

pas, vous avez vraiment besoin d'étudier la science. Vous avez tout simplement

tort.

51 Schneider conteste à nouveau les prétentions scientifiques de son interlocuteur profane. Il conclut sur une nouvelle construction ternaire (moins réussie que la première), annonçant ce qui a été "établi ", et l'associant à une accusation répétée trois fois (" vous vous trompez complètement ») et une invitation réitérée à " étudier la science ".

Et c'est là l'envers de la médaille de l'exercice de l'autorité. Schneider se comporte comme gardien de la communauté scientifique. Lorsqu'un profane tente de parler comme un scientifique, Schneider a le droit de dire qu'il se trompe. Agissant au nom de la communauté scientifique, il peut régenter la frontière entre scientifiques et profanes. Il est possible que le profane s'améliore en passant du temps à « étudier la science »; toujours est-il que d'ici-là, il est réduit au silence.

\section{Résumé et conclusions}

On a vu que les participants à cette rencontre mobilisent deux stratégies principales pour communiquer par-delà l'opposition entre scientifiques et profanes. La première stratégie, dite d'invitation, cherche à combler le fossé en incitant le profane à rejoindre la conversation en cours dans la communauté scientifique. La seconde stratégie d'autorité reconnaît l'existence d'un fossé; le scientifique assume ses responsabilités afin de se montrer crédible - mais en contrepartie, il a le pouvoir de réduire au silence les profanes lorsqu'ils ne se montrent pas dignes de confiance. La première stratégie consiste à identifier les deux groupes l'un à l'autre ; la seconde, à les amener à réaliser une transaction entre eux, négociant les bons comportements communicatifs afin de mériter la confiance.

Dans le contexte de cette émission, la stratégie d'invitation semble l'emporter légèrement sur la seconde stratégie. Schneider réagit à quatre questions de l'auditoire par la production d'éloges, et n'en dénonce que trois comme fautives. Mais il faut noter (ainsi que je l'ai écrit ailleurs, Goodwin et Honeycutt 2009) que même les échanges les plus cordiaux se font sur un fond d'exercice de l'autorité. Ainsi qu'on l'a vu plus haut, implicitement, Schneider joue son rôle dans la transaction autoritaire en manifestant constamment de la modestie dans ses explications. C'est ce qui l'autorise à demander à ses interlocuteurs d'assumer leur propre rôle, soit en lui accordant leur confiance, soit $\mathrm{du}$ moins en s'abstenant de le contredire ouvertement. Tout en louant leur questionnement profane, il se réserve le droit de produire les réponses; droit validé 
par l'animatrice, qui lui accorde des tours de parole plus longs, et lui laisse le dernier mot dans presque tous les échanges. La face sévère de l'autorité apparaît lorsqu'un interlocuteur profane résiste ouvertement à la clôture de l'échange, et dénonce le manque de modestie des réponses de Schneider (" éluder la question ", " alarmiste »). Dans ces cas, l'exercice de l'autorité devient explicite, Schneider mettant fin à la discussion. Ainsi, qu'elle soit exercée explicitement, à travers la possibilité de déclarer que l'autre a tort, ou implicitement, en suscitant la confiance ou en incitant au silence, l'autorité est ce qui permet la clôture des échanges entre scientifiques et profanes - du moins, elle permet les formes de clôture possibles sans que le scientifique en vienne à prendre en charge la formation du profane, jusqu'à l'amener à un niveau d'enseignement supérieur qui permettrait l'identification complète des deux groupes, abolissant ainsi la partition sociale sur laquelle cette analyse s'est ouverte.

Comment le fonctionnement de l'autorité experte, telle qu'elle est comprise par les participants à cette rencontre, s'articule-t-il avec les approches théoriques de l'appel à l'opinion experte? Deux approches principales de l'autorité émergent de la littérature secondaire. La première s'adresse prioritairement aux étudiants, et propose des techniques simples et pratiques pour évaluer les sources d'information. Les manuels et les ressources du même type offrent de longues listes de facteurs à prendre en considération : la source est-elle véritablement experte ? Est-elle récente ? Où est-elle éditée ? Sa bibliographie est-elle complète et à jour? (Ces critères sont identifiés dans Goodwin 2011b.) Les sources d'information vers lesquelles Schneider oriente l'auditoire satisferaient ces critères : les rapports de l'IPCC, l'agence américaine de protection de l'environnement, les sites web universitaires, et, bien entendu, lui-même. Mais lorsqu'on l'invite à dire pourquoi il est digne de foi, il explicite un seul ensemble de facteurs : ceux qu'on a recouverts ici sous le terme de "modestie ». Nombre des aspects d'un comportement communicatif modeste échappent aux listes habituelles de critères de fiabilité d'un expert: l'expression de doutes, d'incertitudes et de limitations, par opposition aux "discours de vérité " et à la "traque aux mythes ». Un autre critère semble en tension avec l'approche académique standard de l'autorité. Celle-ci considère en effet généralement l'existence de désaccords entre experts comme l'indice que les propositions qu'ils avancent ne sont pas fiables; aucun d'eux n'est en mesure d'avancer une réponse incontestable (par ex. Walton 1997). A l'inverse, pour Schneider, reconnaître l'existence de désaccords au sein de la communauté scientifique est un signe de fiabilité, dans la mesure où le fait même d'admettre un fait potentiellement embarrassant montre que le locuteur assume ses responsabilités communicationnelles.

A l'autre bout du spectre, la littérature de recherche propose des reconstructions de la logique qui sous-tend le type d'argument dit "appel à l'opinion experte ». Le travail récent de Wagemans (2011) comporte une utile proposition d'articulation des approches existantes. Partant de la conclusion, à la manière des pragma-dialecticiens, son analyse est la suivante :

1. O [ce que l'expert a dit] est vrai ou acceptable [, parce que:]

1.10 est affirmé par l'expert $\mathrm{E}$ [, ce qui est vrai parce que:]

1.1.1a $E$ est un expert dans le champ $F$ dont relève $O$.

1.1.1b La source $S$ prouve que $O$ est affirmé par $E$.

1.1' [Et] Accepter que $O$ est affirmé par $E$ rend acceptable que $O$ est vrai ou acceptable [, parce que:]

1.10.1a E est personnellement fiable.

1.10.1b E est capable de fournir d'autres preuves de $O$.

1.10.1c 0 est cohérent avec ce que d'autres (types d') experts de $\mathrm{F}$ affirment. 
57 L'importance accordée à la présentation de l'autorité experte dans la discussion analysée témoigne, au minimum, qu'un travail discursif conséquent est nécessaire pour accomplir ce que le théoricien évoque brièvement par l'expression « une assertion par l'expert E», et pour rendre «acceptable » ce qui est dit. Du point de vue des participants, le transfert de la qualité épistémique de l'expert à $\mathrm{O}$ est moins important que la transaction sociale complexe par laquelle $O$ est avancé. Pour reprendre les termes de Collins et Evans, le profane, dans son interaction avec l'expert, s'attache à " produire des jugements sociaux sur à qui manifester son accord, et non des jugements scientifiques sur ce qu'il convient de croire » (2007 : 47-48). Afin de justifier le jugement social du profane, l'expert se plie ostensiblement aux exigences d'une communication responsable en avançant $\mathrm{O}$ - ou, comme je l'ai suggéré ailleurs, l'expert offre une " obligation" ${ }^{8}$ (Goodwin 2001, 2010, 2011a, Goodwin et Dahlstrom 2013). Si le profane estime que cette obligation est solide, alors il fait confiance à l'expert et accepte 0 . Dans le cas contraire, l'expert peut encore se livrer à une forme de chantage : il a le pouvoir de déclarer faux tout propos qui contredit $O$. Dès lors que le profane ne peut affirmer ouvertement son désaccord, la proposition $\mathrm{O}$ avancée par l'expert est traitée comme si elle était acceptée.

58 A l'image des autres ressources sociales que recouvre l'idée d'autorité, l'autorité experte confère du pouvoir à celui qui en jouit dans le même temps qu'elle lui impose des contraintes. Mais ces considérations sont balayées par les reconstructions théoriques standards. Le type d'argument ainsi reconstruit n'est pas erroné pour autant ; mais il laisse échapper ce point important - dont ont pourtant conscience tant les participants qui recourent à ce procédé que ceux qui le subissent.

Toutes les théories de l'argumentation (à l'exception des plus formelles) s'intéressent à des degrés variables à la pratique de l'argumentation - a minima, parce qu'elles peuvent être confirmées ou mises en cause par des observations empiriques. Dans cet article, j'ai cherché à montrer ce qu'une théorie de l'argumentation peut gagner à être loyale envers la conception qu'ont les argumentateurs de ce qu'ils font. J'ai voulu déployer moi-même cette loyauté en observant méthodiquement la mise en œuvre, par les participants, de leur capacité à exercer l'autorité experte et à y répondre par des méthodes plus ou moins explicites, à partir d'une étude de cas particulière. J'ai cherché à organiser des observations disparates dans une restitution plus complète de la discussion, et j'ai entrepris de fonder (ou, mieux peut-être, de trianguler) cette restitution en la mettant en relation avec d'autres cas et d'autres perspectives théoriques 9 .

60 S'agit-il pour moi de proposer une approche purement descriptive de l'étude de l'argumentation? Sur ce point, les traditions francophones et anglophones divergent, les Nord-Américains répondant massivement par un "non", à l'inverse de leurs collègues outre-Atlantique (Doury 2013b). Il se pourrait que cette différence soit en grande partie liée à nos positions socio-professionnelles. Comme de nombreux théoriciens de l'argumentation en Amérique du Nord, je gagne mon pain quotidien en enseignant à de jeunes gens comment devenir de " meilleurs » argumentateurs ; cette entreprise n'a de sens que si on est prêt à admettre qu'être " meilleurs ", eh bien, c'est " mieux ». Plus généralement, à la suite de Robert Craig, je considère la théorie de l'argumentation comme une « discipline pratique », c'est-à-dire

une entreprise intellectuelle - professionnelle relativement cohérente, dont le but essentiel est de cultiver le champ d'une pratique sociale... Une discipline pratique 
«cultive » une pratique en s'engageant, avec la communauté des praticiens, dans un discours réflexif. $\mathrm{Si}$ on considère la réflexion pratique ordinaire comme un processus discursif faisant des allers-retours le long du continuum ThéoriePratique, alors «théorie " (la pensée conceptuelle) et "pratique » (l'action située) peuvent être comprises comme des moments dans ce processus; et une discipline pratique peut être définie comme une entreprise formelle, savante, qui cherche à étendre, faciliter et formaliser ce cycle réflexif de pensée et d'action en entreprenant une étude critique systématique et une reconstruction théorique des pratiques sociales (Craig $1996: 468$ ).

61 Une théorie de l'argumentation peut s'approcher d'un des deux pôles du continuum théorie-pratique, mais elle ne peut lui échapper. Les théoriciens de l'argumentation ne sont donc pas des anthropologues qui feraient du terrain dans une peuplade exotique ; ils sont eux-mêmes des indigènes. Juste des indigènes un peu étranges qui, après des heures passées à des recherches chronophages et après avoir subi des initiations diverses et douloureuses, sont devenus les chamans de l'argumentation.

Cette prétention peut paraître arrogante. Un chaman de l'argumentation a-t-il un statut privilégié par rapport aux autres participants, par exemple sur la controverse autour du changement climatique? Peut-il prendre position (et agir ainsi en militant), et déclarer qui sort vainqueur de la confrontation? Un tel militantisme pose des problèmes pratiques; les cérémonies chamaniques requièrent habituellement pas mal de temps par exemple, et de nombreux débats prennent fin bien avant qu'une sentence soit prononcée ${ }^{10}$. De plus, même en supposant que les interactions argumentatives produisent de bonnes réponses (ce dont je me garderai bien), trouver ces bonnes réponses pourrait bien s'avérer difficile. Comme le reconnaissent les participants euxmêmes, "The Skeptics" constitue juste un moment au sein d'un processus de délibération publique beaucoup plus large sur le changement climatique. Ainsi, le pouvoir de se prononcer avec autorité sur des événements singuliers ne propulse pas les théoriciens de l'argumentation vers des sommets vertigineux. Je pense plutôt que nous jouons des rôles modestes : le premier en cultivant les types d'échanges que nous étudions, et le second en portant un regard curieux sur les interactions argumentatives, avec le désir d'être surpris par ce que nous allons y découvrir.

\section{BIBLIOGRAPHY}

Collins, Harry \& Robert Evans. 2002. « The Third Wave of Science Studies : Studies of Expertise and Experience », Social Studies of Science $32: 2,235-296$

Collins, Harry \& Robert Evans. 2007. Rethinking Expertise (Chicago, IL : University of Chicago Press)

Craig, Robert T. 1996. «Practical-Theoretical Argumentation », Argumentation $10: 4,461-474$

Craig, Robert T. 1999. « Metadiscourse, Theory, and Practice ", Research on Language and Social Interaction $32: 1-2,21-29$ 
Craig, Robert T. 2011. "The Uses of "Argument" in Practical Metadiscourse », Robert C. Rowland (éd.), Reasoned Argument and Social Change (Washington, D.C. : National Communication Association), 76-86

Craig, Robert T. \& Karen Tracy. 2005. " "The Issue" in Argumentation Practice and Theory ", Frans H. van Eemeren \& Peter Houtlosser (éds.), Argumentation in Practice (Amsterdam : John Benjamins), 11-28

Doury, Marianne. 2008. " "Ce n'est pas un argument" : Sur quelques aspects de théorisations spontanées de l'argumentation », Pratiques 139/140, 111-128

Doury, Marianne. 2010a. « Amalgames, procès d'intention et autres scandales argumentatifs ». Conférence présentée lors du colloque International «Communication et Argumentation dans la Sphère Publique », Galati (Roumanie) [En ligne : http://www.academia.edu/5452730/ Amalgames_proc\%C3\%A8s_d_intention_et_autres_scandales_argumentatifs]

Doury, Marianne. 2010b. « Une tentative de dialogue institutionnalisé entre citoyens et experts : Le cas de la conférence des citoyens sur les OGM». Conférence présentée lors du colloque «Communication scientifique, médias et société : Les sciences et les technosciences dans un contexte d'incertitude », Genève [En ligne : http://www.lcp.cnrs.fr/IMG/pdf/ art_doury_geneve.pdf]

Doury, Marianne. 2013a. « Positionnement descriptif, positionnement normatif, positionnement militant », Argumentation et Analyse du Discourse 11, [En ligne : http://aad.revues.org/1540]

Doury, Marianne. 2013b. « The Virtues of Argumentation from an Amoral Analyst's Perspective », Informal Logic $33: 4,486-509$

Goodwin, Jean. 2001. « Cicero's authority », Philosophy \& Rhetoric $34: 1,38-60$

Goodwin, Jean. 2002. « Designing Issues », Frans H. van Eemeren \& Peter Houtlosser (éds), Dialectic and Rhetoric:The Warp and Woof of Argumentation Analysis (Dordrecht : Kluwer), 81-96

Goodwin, Jean. 2007. «What, in Practice, is an Argument? », Hans V. Hansen, Christopher W. Tindale, J. Anthony Blair, Ralph H. Johnson, \& Robert C. Pinto (éds.), Dissensus \& the Search for Common Ground (Windsor, Ontario : Ontario Society for the Study of Argumentation)

Goodwin, Jean. 2010. « Trust in Experts as a Principal-Agent Problem », Chris Reed \& Christopher W. Tindale (éds), Dialectics, Dialogue and Argumentation :An Examination of Douglas Walton's Theories of Reasoning and Argument (London : College Publications), 133-143

Goodwin, Jean. 2011a. « Accounting for the Appeal to the Authority of Experts », Argumentation $25: 3,285-296$

Goodwin, Jean. 2011b. « Accounting for the Force of the Appeal to Authority », Frank Zenker (éd.), Argument Cultures. Proceedings of the 8th International Conference of the Ontario Society for the Study of Argumentation, 1-9

Goodwin, Jean. A paraître. « Climate Scientist Stephen Schneider versusThe Sceptics: A Case Study of Argumentation in Deep Disagreement ", Proceedings of the 8th Conference of the International Society for the Study of Argumentation

Goodwin, Jean \& Michael F. Dahlstrom. 2013. « Communication Strategies for Earning Trust in Climate Change Debates », Wiley Interdisciplinary Reviews : Climate Change 5 : 1, 151-160

Goodwin, Jean \& Lee Honeycutt. 2009. «When Science Goes Public : From Technical Arguments to Appeals to Authority », Studies in Communication Sciences $9: 2,19-30$ 
Kauffeld, Fred J. 2009. « What Are We Learning about the Arguers' Probative Obligations », Scott Jacobs (éd.), Concerning Argument (Washington, D.C. : National Communication Association), 1-31

Martin, Douglas. 2010. « Stephen H. Schneider, climatologist, is dead at 65 », New York Times, 20.7.2010, B16

Philipsen, Gerry. 1992. Speaking Culturally : Explorations in Social Communication (Albany, NY : SUNY Press)

Plantin, Christian. 1996. L'argumentation (Paris : Seuil)

Russill, Chris. 2010. «Stephen Schneider and the 'double ethical bind' of climate change communication », Bulletin of Science, Technology \& Society $30: 1,60-69$

Schneider, Stephen H. 2009. Science as a Contact Sport : Inside the Battle to Save Earth's Climate (Washington : National Geographic)

Snell, Marilyn B. 2009. «TNR Q\&A : Dr. Stephen Schneider », The New Republic, 1-7 [cité d'après http://www.newrepublic.com/article/environment-energy/tnr-qa-dr-stephen-schneider]

Wagemans, Jean H. M. 2011. «The Assessment of Argumentation from Expert Opinion », Argumentation $25: 3,329-339$

Walton, Douglas. 1997. Appeal to Expert Opinion : Arguments from Authority (University Park, PA : The Pennsylvania State University Press)

\section{NOTES}

1. Ainsi, les théories "populaires » des participants sont peu susceptibles d'être touchées par l'inflation dont souffrent les théorisations savantes de l'argumentation, où il semble bien que $\mathrm{N}_{\text {théories }}=\mathrm{N}_{\text {théoriciens. }}$

2. Pour un compte rendu de ce pan de l'activité de Schneider cf. Russill 2010.

3. Les extraits cités correspondent à la transcription de l'émission (Insight SBS, 2010) corrigée sur la base de la vidéo (Insight SBS, 2011). Les membres du public présents sur le plateau sont identifiés par leur nom dans la transcription et dans l'article lorsqu'au moins deux exemples leur sont empruntés. Dans les autres cas, les membres du public sont désignés par "Sc.». L'animatrice Jenny Brockie est désignée par A, et Stephen Schneider, par «SS ». Les accents d'intensité ont été ajoutés à la transcription.

4. C'est Goodwin qui souligne

5. Schneider s'adresse ici à des profanes; il est probable qu'il aurait recouru à davantage de modalisations si d'autres experts avaient été présents sur le plateau (Doury 2010b).

6. Dans la version d'origine: "Because it is in absolutely every single model, it has long been accounted for, and it is completely understood. »

7. Les producteurs ont choisi cette séquence comme bande-annonce de l'émission; ce choix est un bon exemple de la propension des médias à mettre l'accent sur le conflit plus que sur le consensus sur des questions socio-techniques.

8. Au sens financier de «titre de créance »; anglais bond (n.d.t.).

9. Je pense que les théoriciens de l'argumentation peuvent aussi se livrer à une activité critique : ils peuvent critiquer des performances précises qui échoueraient à satisfaire aux normes, ou des pratiques plus générales qui ne mettraient pas en œuvre les normes appropriées. Le cas de Schneider et « The Skeptics " a été choisi comme un exemple supposé de bonne pratique, et je ne pense pas que cette hypothèse ait été contredite par quoi que ce soit dans l'analyse. 
10. Je pense à des adages comme «la chouette de Minerve s'envole au crépuscule », ou «la science va toujours moins vite que la politique» (Collins et Evans 2002).

\section{ABSTRACTS}

The primary loyalty of argumentation theory should be to the practical knowledges arguers are exhibiting and thematizing in ordinary argumentative interactions, since such knowledges make the interactions work. In this essay, I show how theorists can learn from practitioners through a close analysis of the argumentologie populaire that emerged in a televised exchange between eminent scientist/communicator Stephen Schneider and an audience of self-described climate sceptics. In part, Schneider proceeds by inviting his audience to join the scientific community. But both Schneider and his audience recognize the limits of this approach; at base, the question is whether laypeople ought to trust scientists' expertise, and thus also be bound by scientists' authority. I close by pointing out the ways in which participants' understanding of the appeal to expert authority is more sophisticated than the accounts given by argumentation theorists.

Les théoriciens de l'argumentation doivent avant tout rendre justice aux savoirs pratiques que les argumentateurs déploient et thématisent dans les interactions argumentatives ordinaires, puisque ce sont précisément ces savoirs qui rendent les interactions possibles. Dans cet article, je montre que les théoriciens peuvent apprendre des praticiens à travers l'analyse minutieuse de l'«argumentologie populaire» qui a émergé au cours d'échanges télévisés entre Stephen Schneider, scientifique et communicant éminent, et un public constitué de "climatosceptiques ", comme ils se désignent eux-mêmes. Une des stratégies mobilisées par Stephen Schneider consiste à inviter le public à rejoindre la communauté scientifique. Mais aussi bien Schneider que le public reconnaissent les limites de cette approche ; la question de base est celle de la confiance : les profanes sont-ils disposés à faire confiance à l'expertise des scientifiques, et faire ainsi allégeance à leur autorité ? L'article conclut sur la suggestion que la façon dont les participants comprennent l'appel à l'autorité experte est plus sophistiquée que les descriptions qu'en proposent les théoriciens de l'argumentation.

\section{INDEX}

Keywords: appeal to expert authority, argumentation, expertise, metadiscourse, science communication, science-policy interface

Mots-clés: appel à l'autorité experte, argumentation, communication de la science, expertise, interface science-société, métadiscours

\section{AUTHORS}

\section{JEAN GOODWIN}

Iowa State University 